\title{
Evaluation of tradipitant, a selective NK1 antagonist, on response to oxycodone in humans
}

\author{
Marion A. Coe ${ }^{1,2,3} \cdot$ Michelle R. Lofwall $^{1,4,5} \cdot$ Victoria Vessels $^{1}$ • Paul A. Nuzzo ${ }^{1}$ • Sharon L. Walsh ${ }^{1,2,4,5,6}$ \\ Received: 7 January 2021 / Accepted: 1 March 2021 / Published online: 14 May 2021 \\ (C) The Author(s), under exclusive licence to Springer-Verlag GmbH Germany, part of Springer Nature 2021
}

\begin{abstract}
Rationale Preclinical studies demonstrate that the NK1 receptor is involved in opioid reinforcement and withdrawal expression. Few studies have examined the impact of treatment with NK1 antagonists on opioid response in humans.

Objective To explore the potential for a selective NK1 antagonist, tradipitant, to attenuate the abuse liability and reinforcing and analgesic effects of oxycodone in opioid-experienced individuals.

Methods Participants with recreational opioid use, but without opioid physical dependence, were enrolled as inpatients for $\sim 6$ weeks $(n=8)$. A within-subject, double-blind, randomized, placebo-controlled, crossover design was employed. The pharmacodynamic response to intranasal oxycodone across a range of doses (0 to $30 \mathrm{mg}$ ) was examined during two counterbalanced maintenance periods (tradipitant 0 or $85 \mathrm{mg} /$ bid). Oxycodone self-administration was assessed with a modified progressive ratio procedure, and analgesia was assessed with the cold pressor test.

Results Oxycodone produced significant and dose-related increases on a broad array of prototypic opioid measures, including subjective ratings related to abuse liability (e.g., liking) and physiological outcomes (i.e., expired $\mathrm{CO}_{2}$ ). Oxycodone selfadministration increased with increasing dose, as did analgesia. Tradipitant largely did not alter any of these effects of oxycodone, with the exception of producing a reduction in ratings of desire for opioids.

Conclusions Given that the vast majority of oxycodone effects were unchanged by tradipitant, these data do not provide support for the utility of NK1 antagonists as a potential treatment for opioid use disorder.
\end{abstract}

Keywords Oxycodone $\cdot$ Opioid $\cdot$ Tradipitant $\cdot$ NK1 antagonist $\cdot$ Self-administration $\cdot$ Abuse liability $\cdot$ Human

Sharon L. Walsh

sharon.walsh@uky.edu

1 Center on Drug and Alcohol Research, University of Kentucky, 845 Angliana Avenue, Lexington, KY 40508, USA

2 Department of Pharmacology, University of Kentucky, 845 Angliana Avenue, Lexington, KY 40508, USA

3 Present address: Pinney Associates, Inc., 4800 Montgomery Ave, Suite 400, Bethesda, MD 20814, USA

4 Behavioral Science, University of Kentucky, 845 Angliana Avenue, Lexington, KY 40508, USA

5 Psychiatry, University of Kentucky, 845 Angliana Avenue, Lexington, KY 40508, USA

6 Pharmaceutical Sciences, University of Kentucky, 845 Angliana Avenue, Lexington, KY 40508, USA

\section{Introduction}

The neurokinin-1 (NK1), or substance $\mathrm{P}$, receptor is a $\mathrm{G}$ protein-coupled receptor distributed throughout the central and peripheral nervous systems with high concentrations in the spinal cord and reward- and affect-related brain regions (e.g., nucleus accumbens). Highly selective NK1 receptor antagonists were developed and investigated for therapeutic indications targeting pain, vomiting, anxiety, depression, pruritis, and alcohol use disorder; but as yet, the only FDAapproved indication for an NK1 receptor antagonist is chemotherapy-induced nausea and vomiting with several products now marketed for this purpose.

Preclinical studies in the 1990s and 2000s consistently demonstrated that the NK1 receptor system was highly involved in opioid reward, drug seeking, withdrawal, and physical dependence; these effects were observed across an array of laboratory models and species. For example, in NK1 knockout mice, morphine reward, as measured by conditioned 
place preference (CPP), is absent, and self-administration of morphine is attenuated (Murtra et al. 2000). In contrast, NK1 knockout mice develop CPP to food and cocaine, suggesting that deletion of the NK1 receptor selectively reduces opioid reward (Murtra et al. 2000). Similarly, when chronically treated with an NK1 antagonist, self-administration of heroin was decreased in rats trained with both short (1 h) and long (12 h) access to heroin (Barbier et al. 2013). Finally, depression of intracranial self-stimulation (ICSS) thresholds by morphine was attenuated by pharmacological blockade of the NK1 receptor (Robinson et al. 2012). The evidence for the NK1 receptor system's role in opioid physical dependence and withdrawal comes from studies with NK1 knockout mice. When spontaneously withdrawn from morphine after chronic exposure, NK1 knockout mice exhibit fewer signs of opioid withdrawal than wildtype control (Murtra et al. 2000). In addition, NK1 knockout mice chronically exposed to morphine to induce physical dependence do not develop conditioned place aversion to naloxone (Murtra et al. 2000).

Given the strong preclinical evidence for the therapeutic potential of NK1 receptor antagonism in treating symptoms of opioid use disorder, we previously investigated the effect of NK1 antagonism on abuse-related effects of opioids in persons with recreational opioid use but without opioid physical dependence (Walsh et al. 2013). In that study, the only FDAapproved NK1 receptor antagonist at the time, aprepitant (Emend®), was administered alone and with oxycodone. Alone, aprepitant produced no signals of abuse, but it significantly enhanced, rather than reduced, ratings of oxycodoneinduced euphoria and drug liking. Some objective responses to oxycodone (e.g., respiratory function, observer-rated opioid agonist effects) were similarly enhanced by pretreatment with aprepitant. A subsequent study, which enrolled individuals maintained on methadone, examined the effects of aprepitant versus placebo given preceding the usual methadone dose and similarly reported that aprepitant enhanced the subjective response to methadone (Jones et al. 2013).

There are at least two possible explanations for these clinical results, which contrast so distinctly from the preclinical studies. First, aprepitant, oxycodone, and methadone are all primarily metabolized by liver cytochrome P4503A4. Importantly, aprepitant is a known potent inhibitor and inducer of P4503A4, and prior clinically meaningful drug-drug interactions have been reported (Majumdar et al. 2003; Merck \& Co. 2021; Sanchez et al. 2004). Thus, the finding of enhanced response to oxycodone in the presence of aprepitant may be attributable to a pharmacokinetic interaction in which oxycodone concentrations were increased (leading to increased pharmacodynamic effects). Because blood samples were not collected during these studies, this hypothesis could not be verified. The second possible explanation could be that the aprepitant dose was too low to reach sufficient NK1 receptor occupancy. NK1 receptor occupancy by aprepitant is dose- dependent, and at doses exceeding what was used in the clinical abuse potential studies, $90 \%$ central receptor occupancy was achieved (Hargreaves 2002). Both aprepitant and another NK1 antagonist, casopitant, demonstrated efficacy for depression in clinical trials when administered at doses that resulted in near-complete central receptor occupancy (Ratti et al. 2011; Zamuner et al. 2012). Collectively, these data suggest that high central receptor occupancy may be critical for efficacy of NK1 antagonists to produce behavioral effects. Animal data show that NK1 receptor antagonism promotes substance $\mathrm{P}$ release, ostensibly via blockade of presynaptic autoreceptors (Singewald et al. 2008). Thus, unless sufficient occupancy of postsynaptic receptors is achieved, the enhanced release of substance P may exhibit paradoxical effects.

The current study examined the effect of a different NK1 antagonist, tradipitant (Vanda Pharmaceuticals, VLY686, and previously Lilly, LY686017), on response to opioids in humans. Tradipitant is an investigational drug that is currently under evaluation for efficacy in gastroparesis, COVID-19induced pneumonia, motion sickness, and pruritis in atopic dermatitis. Tradipitant was selected for investigation because it is highly selective for the NK1 receptor, is not reported to produce drug-drug interactions via CYP4503A4, and has been shown to achieve $93 \pm 4 \%$ receptor occupancy in the human frontal cortex following steady-state dosing of $100 \mathrm{mg}$ daily (Tauscher et al. 2010). The study design allowed for evaluation of the interaction between tradipitant and oxycodone after acute pretreatment with tradipitant and after steady-state concentrations were achieved on a broad array of abuse-related subjective responses, physiological responses, and analgesic responses in individuals with sporadic recreational opioid use.

\section{Materials and methods}

\section{Participants}

Participants were healthy adults with ages $18-50$ who reported sporadic current illicit opioid use (confirmed by at least one opioid positive urine toxicology test during screening), at least 1 year of illicit use and prior use by the intranasal route of administration. An opioid negative urine sample was also required during screening in the absence of withdrawal signs to preclude opioid physiological dependence. Good health was determined by medical history, psychiatric and physical evaluation, an ECG, nasal exam, and blood and urine chemistries with no clinically significant findings. Exclusion criteria included (1) seeking substance use treatment or successfully sustaining abstinence in the community; (2) pregnant or breastfeeding; (3) BMI > 30; (4) history of seizure disorders, asthma or other respiratory disorders, head injury, hypertension, cardiovascular disease, active and clinically significant liver disease, and abnormal electrocardiogram (ECG); or (5) 
physical dependence on any drug requiring medical detoxification (i.e., alcohol, benzodiazepines). Participants were recruited through flyers, newspaper, and magazine advertisements and by word-of-mouth. The study was approved by the University of Kentucky (UK) Institutional Review Board and the Food and Drug Administration, conducted in accordance with the Declaration of Helsinki, and participants gave written informed consent. A Certificate of Confidentiality was obtained from the National Institute on Drug Abuse, and volunteers were paid for their participation.

\section{Study design and setting}

This $~ 6.5$-week inpatient study used a randomized, placebocontrolled, within-subject, crossover design and took place on a residential research unit within a hospital. After admission, participants were trained on study procedures and completed an active training session (see description below). Thereafter, the study commenced with initiation of maintenance dosing on tradipitant at either 0 or $85 \mathrm{mg}$, bid (8:30 AM and 8:30 PM) with the order randomized and counterbalanced across participants. Assigned doses were administered daily for $\sim 16$ days (period 1). This was followed by a 5-day washout period before participants were crossed over to the alternate dose condition for an additional $\sim 16$ days (period 2 ). Eight experimental sessions were conducted ( 2 cumulative challenge sessions and 3 sets of paired sample and self-administration sessions) during each maintenance period (see details below and Fig. 1 for study design schema).

\section{Study drugs}

This study was conducted under an investigator-initiated investigational new drug application from the Food and Drug Administration (\#130,940). Tradipitant (85 mg) and its matched placebo capsules were obtained from Vanda Pharmaceuticals (Washington D.C., USA). Commercially available oxycodone $\mathrm{HCl}$ powder (Mallinckrodt, Hazelwood, MO) and diluent (lactose monohydrate powder;
Medisca Pharmaceuticals, Plattsburgh, NY) were used for intranasal test doses. IN doses were formulated in identical volumes (for blinding purposes) by the addition of lactose as needed. For IN administration, volunteers were instructed to split the powder into two lines and insufflate one line through each nostril using a straw.

\section{Experimental test sessions}

Training/qualification sessions Two training/qualification sessions $(2.5 \mathrm{~h}$ each) were conducted in a single day (8:00 AM and 1:00 PM) to test participant response to 0 - and $15-\mathrm{mg}$ oxycodone (doses administered in randomized order and counterbalanced across participants). Participants unable to discriminate between placebo and active drug are discharged from the study.

General experimental methods Experimental sessions were conducted at the same time of day beginning at 9:00 AM, with $30 \mathrm{~min}$ of baseline data collection. Experimental sessions were timed so that the peak effects of the morning tradipitant dose were achieved during the session. During all test sessions, computerized questionnaires were completed by both the participant and a trained observer, and physiological data were collected (see Table 1 for timeline). Urine toxicology was performed daily to test for unauthorized illicit drug use (including cocaine, THC, amphetamine, methamphetamine, methadone, opiates, barbiturates, and benzodiazepines); females were tested weekly for pregnancy. A caffeine-free diet of three standard meals daily and snacks available between meals was provided. Cigarette smoking and food were restricted just prior to test sessions for 60 and $90 \mathrm{~min}$, respectively. Certain medications (e.g., acetaminophen, magnesia, and ibuprofen) were available to volunteers as needed, but administration was restricted after midnight preceding sessions and during test sessions. A nasal exam was conducted by nursing staff before and after each test session to assess occlusion or injury.
Fig. 1 The study design timeline is shown illustrating the two periods of tradipitant dosing in this crossover study ( 0 or $85 \mathrm{mg}$ / bid) and the timing of all experimental test sessions

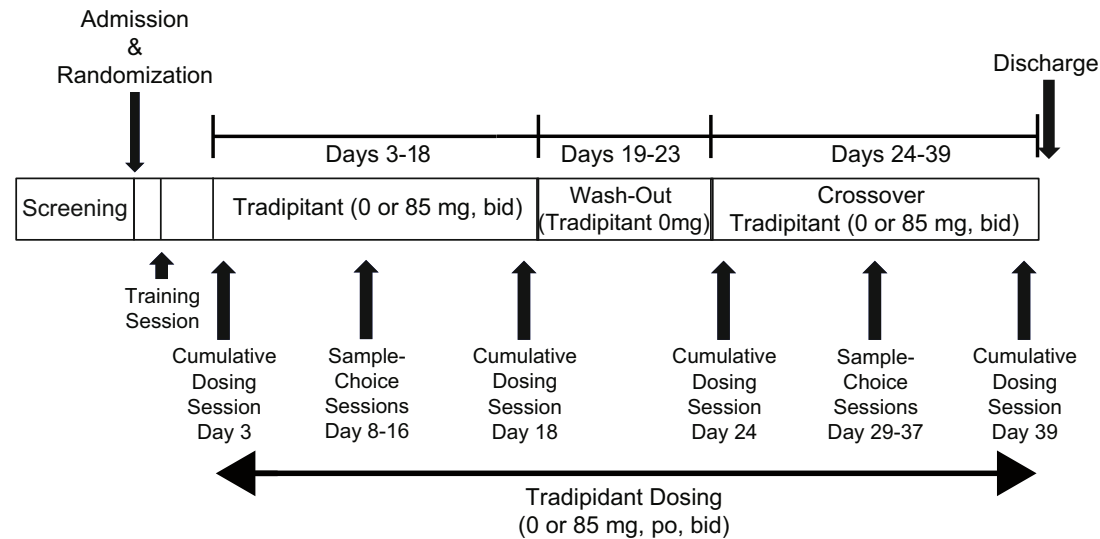


Table 1 Study timeline for data collection for each of the three types of experimental sessions

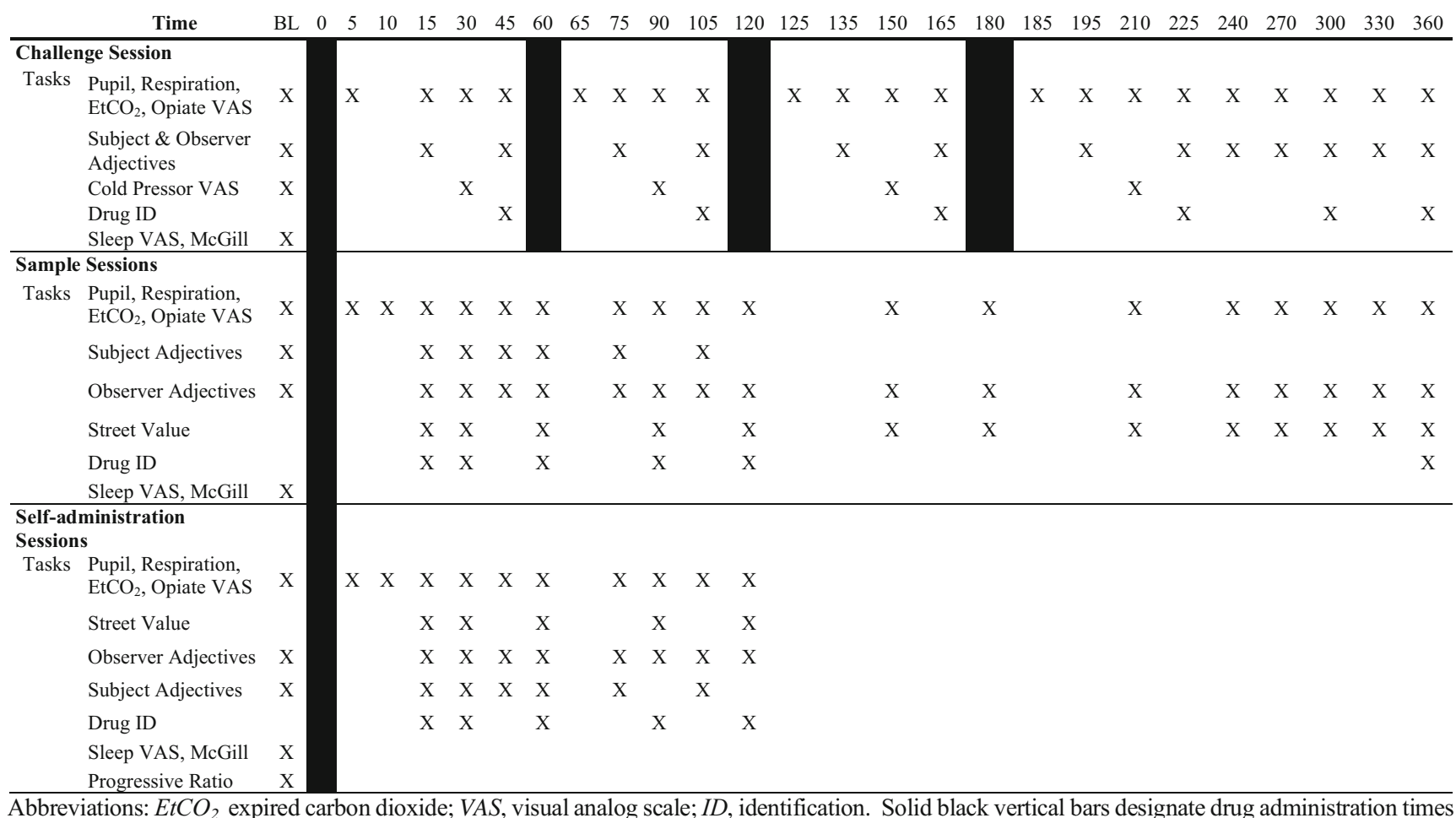

Oxycodone cumulative dosing sessions During each treatment period, two oxycodone cumulative dosing sessions were conducted during which oxycodone was administered at $0,5,10$, and $20 \mathrm{mg}$ in ascending order at 1 -h intervals (i.e., cumulative doses of $0,5,15$, and $35 \mathrm{mg}$ ). These occurred on day 1 of dosing (to examine the acute interaction) and on day 14 after steady-state was achieved $\left(\mathrm{t}_{1 / 2}\right.$ estimated at $16 \mathrm{~h}$ for tradipitant (Tauscher et al. 2010)). Data were collected for $3 \mathrm{~h}$ after the last oxycodone administration.

Sample and self-administration sessions Sample and selfadministration sessions were conducted in pairs. Oxycodone $(0,15$, or $30 \mathrm{mg} / 70 \mathrm{~kg}$, IN, randomized order) was given during the sample session, and participants were told they could work for that same dose during the self-administration session the following day. For sample sessions, data were collected for $6 \mathrm{~h}$ after drug administration. For selfadministration sessions, participants were given the opportunity to work (i.e., button pressing on the computer mouse) for 7 consecutive trials to earn the dose they sampled the preceding day or money over $2 \mathrm{~h}$. The amount of required work increased with successive trials using a progressive ratio schedule (i.e., 50, 250, 500, 1000, 1500, 2000, and 2500 responses). The number of responses (with a programmed interresponse interval of $0.6 \mathrm{~s}$ ) was displayed on the computer monitor until the response requirement was met or time had expired. During each trial, participants could work for $1 / 7$ th of the total sample dose, for US $\$ 3$, or choose not to work. The schedules for money and drug were concurrent and advanced through the ratio requirements independently of one another. Participants could receive all or a fraction of the dose, a combination of drug and money, and only money (totaling \$21 if money was exclusively chosen), which were delivered immediately after responding was completed.

\section{Subject- and observer-rated measures}

Visual analog scales (VAS) to assess opioid effects rated from 0 ("not at all") to 100 ("extremely") included the following: Do you feel any DRUG EFFECT? How HIGH are you? Does the drug have any GOOD effects? Does the drug have any BAD effects? How much do you LIKE the drug? How much do you DESIRE OPIATES right now? During the cumulative dose sessions only, additional VAS were used to assess the response to the cold pressor test (How PAINFUL was the sensation you just experienced? How UNPLEASANT was the sensation you just experienced? How BOTHERSOME was the sensation you just experienced?). A 17-item adjective checklist scored 0 ("not at all") to 4 ("extremely") encompassing the opioid agonist and Fraser scales (Fraser et al. 1961; Preston et al. 1987), pharmacological class questionnaire, and street value questionnaire along with an observer-rated adjective scale that was completed by a trained research assistant (Walsh et al. 2008) were used during sessions. 


\section{Physiological measures}

Oxygen saturation, pulse, and resting blood pressure were collected continuously (Dinamap Non-invasive Patient Monitor; GE Medical Systems, Tampa, FL, USA) for $30 \mathrm{~min}$ before and up to $6 \mathrm{~h}$ after drug administration. Pupil diameter under constant light conditions (NeurOptics Pupillometer; San Clemente, CA, USA), respiratory rate, and end-tidal $\mathrm{CO}_{2}$ (Capnograph N85; Nellcor, Boulder, $\mathrm{CO}$, USA) were collected at regular intervals (see Table 1).

\section{Cold-pressor task (CPT)}

The CPT was conducted during each oxycodone cumulative dosing session at baseline and $30 \mathrm{~min}$ after each drug administration. Two water coolers (one containing roomtemperature water $\left[24.0 \pm 0.5^{\circ} \mathrm{C}\right]$ and one containing cold water $\left.\left[1.0 \pm 0.5^{\circ} \mathrm{C}\right]\right)$ were used; the latter employed an aquarium pump to maintain consistent water temperature. Participants submerged their nondominant arm above the elbow into the room-temperature water for $2 \mathrm{~min}$ and then immediately transferred their arm to the cold water. They verbally reported the moment they first felt pain (i.e., pain threshold) and when they could no longer tolerate the pain and removed their arm from the water (i.e., pain tolerance); these outcomes were measured in seconds. For safety reasons, the maximum time an arm could be submerged in the cold bath was $5 \mathrm{~min}$.

\section{Safety assessments}

A checklist querying side effects common to NK1 antagonists, extracted from the tradipitant investigator brochure, was completed by participants each night. Blood samples were drawn to assess liver function (AST, ALT, alkaline phosphatase, and total bilirubin) throughout the study on admission and after 5 and 13 days of dosing in each Treatment Period.

\section{Statistical analysis}

Demographics, side effects, and drug use characteristics are reported descriptively. Physiological measures collected every minute were averaged across time ( $5-30 \mathrm{~min})$ corresponding to collection of subjective reports. All physiological, subjective-, and observer-rated measures were initially analyzed as raw time course data followed by peak $\left(E_{\min }\right.$ or $E_{\max }$ ) scores analyses. Sample session time course models included three factors, tradipitant dose (2 levels), oxycodone dose (3 levels), and time. Time course analyses for the cumulative dosing session included four factors, tradipitant dose (2 levels), initiation/steady-state (2 levels), oxycodone dose (4 levels), and time. For the peak analyses, the factor of time was removed; otherwise, the models remained the same. Self- administration session models included the factors of tradipitant dose (2 levels) and oxycodone dose (3 levels). Tukey post hoc tests were performed to explore main effects and interactions. Analyses were run with Proc Mixed in SAS 9.3 (SAS Institute, Inc.; Cary, NC, USA), which is suited for data with repeated measures, correlations among observations, and missing data. Results were considered significant when $p \leq 0.05$ and means (standard errors) are reported unless otherwise indicated.

\section{Results}

\section{Participants}

Of the 40 individuals who were screened, 28 failed to pass the inclusion/exclusion screening, 13 were enrolled (11 male, 2 female), and 8 completed the study. Of those who completed, the average age was 34 years old, all were male, their average history of illicit opioid use was $\sim 12.6$ years, and, while intranasal opioid use was an inclusion criteria, 5 of the 8 also reported intravenous opioid use. Seven volunteers met past year DSM 5 criteria for opioid use disorder (ranging from mild to severe). Polysubstance use was common among this sample with some individuals meeting past year DSM 5 criteria for other substance use disorders, including methamphetamine $(n=1)$, cannabis $(n=1)$, cocaine $(n=2)$, and alcohol $(n=$ $3)$. Of the completers, one-half received placebo maintenance first, and one-half received active tradipitant first before crossing over to the opposite other condition.

\section{Sample sessions}

\section{Subject-rated outcomes}

As shown in Fig. 2, oxycodone significantly $(p<.05)$ increased VAS ratings for drug liking, good effects, and overall drug effect in a dose-dependent manner (see figure legends for statistical outcomes). Tradipitant did not significantly alter these responses to oxycodone. In contrast, trough ratings of VAS desire opiates were not significantly altered by oxycodone, but there was a main effect of tradipitant $(p<.05)$, whereby ratings of desire for opioids were lower during maintenance on tradipitant compared to placebo across all oxycodone doses, including placebo (Fig. 2). Oxycodone also significantly increased ratings on the opioid agonist-scale and numerous individual-scale items, as well as ratings of the street value of the drug (see Table 2 for outcomes not depicted graphically). However, again tradipitant did not significantly alter any of these outcomes. 
Fig. 2 Mean peak visual analog ratings $(n=8 ; \pm 1$ SEM) are displayed as a function of tradipitant and oxycodone dose. These data were collected during the sample sessions. Significant main effects of oxycodone dose were found for $E_{\max }$ for drug liking (upper left: $\mathrm{F}_{(2,14)}=24.5$, $p<0.0001$ ), good effects (upper right: $\left.\mathrm{F}_{(2,14)}=30.8, p<0.0001\right)$, and any drug effect (lower left; $\left.\mathrm{F}_{(2,14)}=35.6 p<0.0001\right)$. There was a main effect of tradipitant on ratings for desire for opiates $\left(E_{\min }\right)$ (lower right; $\mathrm{F}_{(1,7)}=5.7$, $p=0.048)$, but no significant post hoc differences. Asterisks (*) indicate a significant difference from the placebo within tradipitant condition (e.g., $0 \mathrm{mg}$ vs. 15 within the placebo tradipitant condition) (Tukey post hoc, $p<0.05$ )
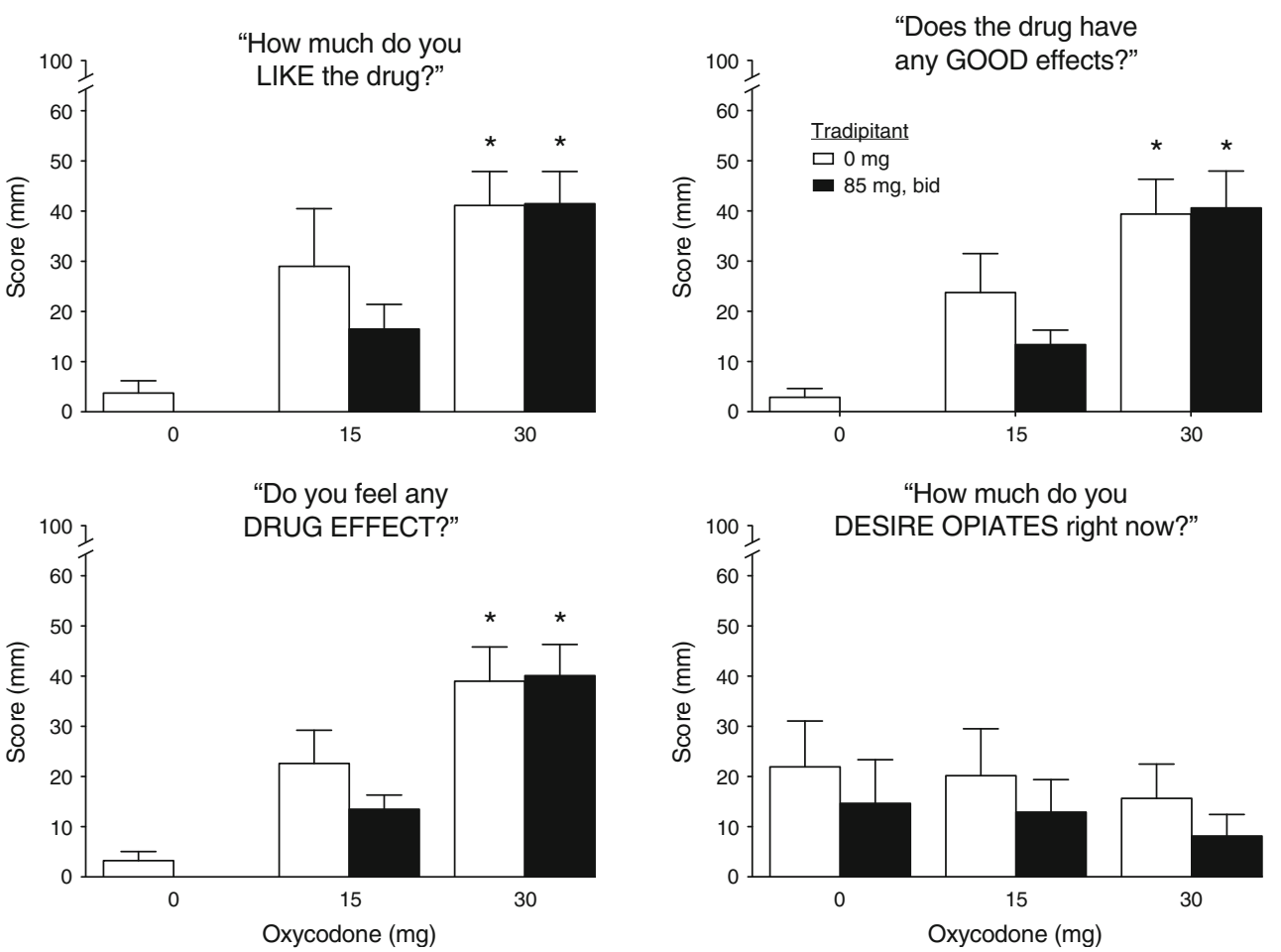

\section{Observer-rated outcomes}

Observer ratings on adjective scales assessing opioid agonist effect were both time- and dose-dependent with significant increases after oxycodone administration compared to placebo (Table 2). There were significant dose effects for ratings on the agonist scale overall and the scale items of skin itchy, nodding, relaxed, coasting, talkative, heavy/sluggish, sleepy, good mood, and energetic. However, there were no significant main effects of tradipitant.

\section{Physiological outcomes}

Compared to placebo, oxycodone produced significant timeand dose-dependent miosis as expected (Fig. 3). Oxycodone also produced significant decrements in measures related to respiratory function, including decreased respiratory rate and oxygen saturation (Fig. 3) and increased end-tidal $\mathrm{CO}_{2}$ $(p<.05$ for all). However, no main effects for or interactions with tradipitant were observed. Additionally, the time course analyses revealed significant main effects of time for heart rate $(\mathrm{F}[18,126]=14.5)$, systolic $(\mathrm{F}[8,126]=3.6)$ and diastolic blood pressure $(\mathrm{F}[18,126]=3.2)(p<.0001$; data not shown $)$, with each decreasing over the course of session for all dosing conditions but no main effects of oxycodone or tradipitant. There were no significant main effects of tradipitant or interactions with tradipitant for any physiological outcome measure.

\section{Self-administration outcomes}

Figure 4 illustrates the pattern of findings for the four related self-administration outcomes: the number of trials worked for drug, the number of trials worked for money, total money earned/session, and the number of trials during which the participant chose not to work. As shown, trials working for drug significantly increased with increasing oxycodone dose, while conversely trials working for money or choosing not to work decreased dose dependently. Tradipitant maintenance had no effect on any self-administration outcome (i.e., trials worked for drug or money, money earned, trials not worked).

\section{Cumulative dosing sessions}

The majority of subjective, observer-rated, and physiological measures collected during the cumulative dosing sessions are not reported here because those results largely align with the pattern of findings obtained in and reported for the sample sessions (see above), whereby oxycodone produced a prototypical opioid agonist profile and tradipitant did not alter this. There were, however, a few exceptions. Similar to the finding for reduced desire during the sample sessions, VAS ratings of desire opiates were altered by tradipitant. Comparison of oxycodone response on day 1 of tradipitant dosing to data obtained after tradipitant steady-state was achieved, and ratings of desire opiates were significantly reduced $(\mathrm{F}[1,7]=6.4$; $p=.030$ ). Additionally, there was a significant tradipitant dose 
Table 2 Data are shown for outcomes from the sample sessions only for which significant effects of oxycodone were found (means (SEM)); data depicted elsewhere graphically are not included

\begin{tabular}{|c|c|c|c|c|c|c|c|}
\hline & & \multicolumn{6}{|c|}{ Oxycodone (mg IN) } \\
\hline & \multirow[b]{3}{*}{$F(2,14)$} & \multicolumn{2}{|r|}{0} & \multicolumn{2}{|r|}{15} & \multicolumn{2}{|c|}{30} \\
\hline & & \multicolumn{6}{|c|}{ Tradipitant (mg p.o./bid) } \\
\hline & & 0 & 85 & 0 & 85 & 0 & 85 \\
\hline \multicolumn{8}{|l|}{ Maximum Peak Values } \\
\hline \multicolumn{8}{|l|}{ Subjective Outcomes } \\
\hline VAS High & 34.82 & $3.38(2.10)$ & $0.00(0.00)$ & $22.00(6.89)$ & $12.25(3.12)$ & $39.75(7.09)$ & $36.88(5.82)$ \\
\hline Street Value (\$) & 41.31 & $0.86(0.64)$ & $0.00(0.00)$ & $14.75(3.05)$ & $13.88(2.57)$ & $25.44(4.86)$ & $26.13(5.70)$ \\
\hline Adjective Agonist Scale & 38.71 & $6.75(1.53)$ & $7.13(1.39)$ & $12.00(1.46)$ & $11.75(1.97)$ & $17.75(1.90)$ & $19.25(3.20)$ \\
\hline \multicolumn{8}{|l|}{ Observer-rated Outcomes } \\
\hline Adjective Agonist Scale & 17.23 & $4.63(0.46)$ & $4.75(0.53)$ & $7.63(0.73)$ & $6.88(0.77)$ & $9.63(0.96)$ & $11.38(2.01)$ \\
\hline Skin Itchy & 21.63 & $0.00(0.00)$ & $0.00(0.00)$ & $0.63(0.26)$ & $0.50(0.27)$ & $1.25(0.25)$ & $1.63(0.32)$ \\
\hline Nodding & 4.22 & $0.00(0.00)$ & $0.00(0.00)$ & $0.38(0.18)$ & $0.00(0.27)$ & $0.38(0.38)$ & $0.88(0.40)$ \\
\hline Relaxed & 9.02 & $1.00(0.00)$ & $1.00(0.00)$ & $1.63(0.26)$ & $0.00(0.00)$ & $1.88(0.23)$ & $2.00(0.27)$ \\
\hline Coasting & 7.12 & $0.25(0.25)$ & $0.00(0.00)$ & $0.25(0.16)$ & $0.00(0.00)$ & $0.63(0.38)$ & $1.00(0.33)$ \\
\hline Talkative & 12.66 & $0.75(0.25)$ & $0.63(0.26)$ & $1.50(0.19)$ & $1.50(0.19)$ & $1.63(0.26)$ & $1.88(0.35)$ \\
\hline Heavy/Sluggish & 6.16 & $0.00(0.00)$ & $0.25(0.16)$ & $0.75(0.37)$ & $0.00(0.00)$ & $0.75(0.41)$ & $1.00(0.33)$ \\
\hline Sleepy & 7.49 & $0.38(0.18)$ & $0.88(0.30)$ & $1.00(0.38)$ & $0.50(0.27)$ & $1.38(0.46)$ & $1.50(0.42)$ \\
\hline Good Mood & 8.93 & $0.88(0.13)$ & $1.00(0.00)$ & $1.25(0.16)$ & $1.63(0.18)$ & $1.63(0.18)$ & $1.75(0.31)$ \\
\hline Energetic & 5.63 & $0.25(0.16)$ & $0.00(0.00)$ & $0.75(0.16)$ & $0.50(0.19)$ & $0.75(0.25)$ & $0.88(0.35)$ \\
\hline \multicolumn{8}{|l|}{ Physiological Outcomes } \\
\hline Systolic BP (peak) & 4.07 & $115.95(3.25)$ & $117.06(1.85)$ & $120.25(4.37)$ & $120.89(2.22)$ & $122.19(3.15)$ & $121.84(3.52)$ \\
\hline Heart Rate (trough) & 6.09 & $61.80(3.13)$ & $60.79(3.52)$ & $57.60(2.85)$ & $58.88(2.57)$ & $56.93(3.06)$ & $57.14(1.98)$ \\
\hline Mean Arterial BP (peak) & 3.85 & $86.36(2.06)$ & $87.75(2.05)$ & $87.75(2.05)$ & $87.45(1.50)$ & $88.97(1.75)$ & $88.94(2.18)$ \\
\hline Oxygen Saturation (trough) & 26.76 & $97.03(0.26)$ & $97.55(0.24)$ & $96.19(0.31)$ & $96.38(0.19)$ & $95.51(0.42)$ & $95.74(0.37)$ \\
\hline
\end{tabular}

Bolded values indicate a significant difference between placebo and active oxycodone within tradipitant dose condition

by time of challenge $(\mathrm{F}[1,7]=6.8 ; p=.036)$, whereby pupils were consistently smaller after steady-state was achieved compared to the first day of tradipitant dosing after IN challenge with $0-, 5-, 10-$, and 20-mg oxycodone (a similar reduction can be noticed in Fig. 3, but that failed to reach significance). Another cluster of significant findings were for measures related to subjective ratings of sedation, including sleepy $(\mathrm{F}[1,7]=14.6 ; p=.007)$ and heavy/sluggish feeling $(\mathrm{F}[1,7]=8.7 ; p=.022)$, whereby the data show that tradipitant generally increased these ratings modestly compared to placebo or ratings increased as a function of days on tradipitant.

A unique contribution of the cumulative dosing sessions is the cold-pressor test assessing pain and analgesia. All behavioral outcomes (time-to-pain threshold, time-to-pain tolerance; see Fig. 5) and subjective VAS outcomes of pain reporting (i.e., painful and intense [Fig. 5] and unpleasant and bothersome [Table 2]) were significantly altered in a dose-dependent fashion by oxycodone, with threshold and tolerance increasing and subjective reports of pain decreasing. However, there was no evidence that either acute or chronic dosing with tradipitant modulated the subjective response to pain or analgesic response to oxycodone.

\section{Safety outcomes}

Tradipitant was generally well-tolerated. Four of the nine side effect checklist symptoms queried were reported more often during the tradipitant maintenance compared to placebo (i.e., fatigue, muscle soreness, dry mouth, and headache). Fatigue and headache were the symptoms reported by the greatest percentage of participants during tradipitant maintenance (both reported by one-third of participants). Tradipitant had no effect on monitored liver function test results (i.e., AST, ALT, or bilirubin). One volunteer exhibited significantly elevated liver function tests approximately halfway through the study, and they were discharged from the study after further labs and were subsequently diagnosed with hepatitis $\mathrm{C}$. They had been randomized to placebo maintenance first and had not yet been exposed to tradipitant. 
Fig. 3 Mean $(n=8 ; \pm 1$ SEM $)$ peak ratings are displayed as a oxycodone dose with data collected during the sample sessions. Significant main effects of oxycodone were found for $E_{\text {min }}$ pupil diameter $\left(\mathrm{F}_{(2,14)}=93.5, p<\right.$ $0.0001), E_{\min }$ respiratory rate $\left(\mathrm{F}_{(2,14)}=4.4, p=0.03\right)$, and $E_{\max }$ end tidal $\mathrm{CO}_{2}\left(\mathrm{~F}_{(2,14)}=20.5 p<\right.$ $0.001)$. Asterisks (*) indicate a significant difference from the placebo within tradipitant condition (Tukey post hoc, $p<$ $0.05)$ function of tradipitant and
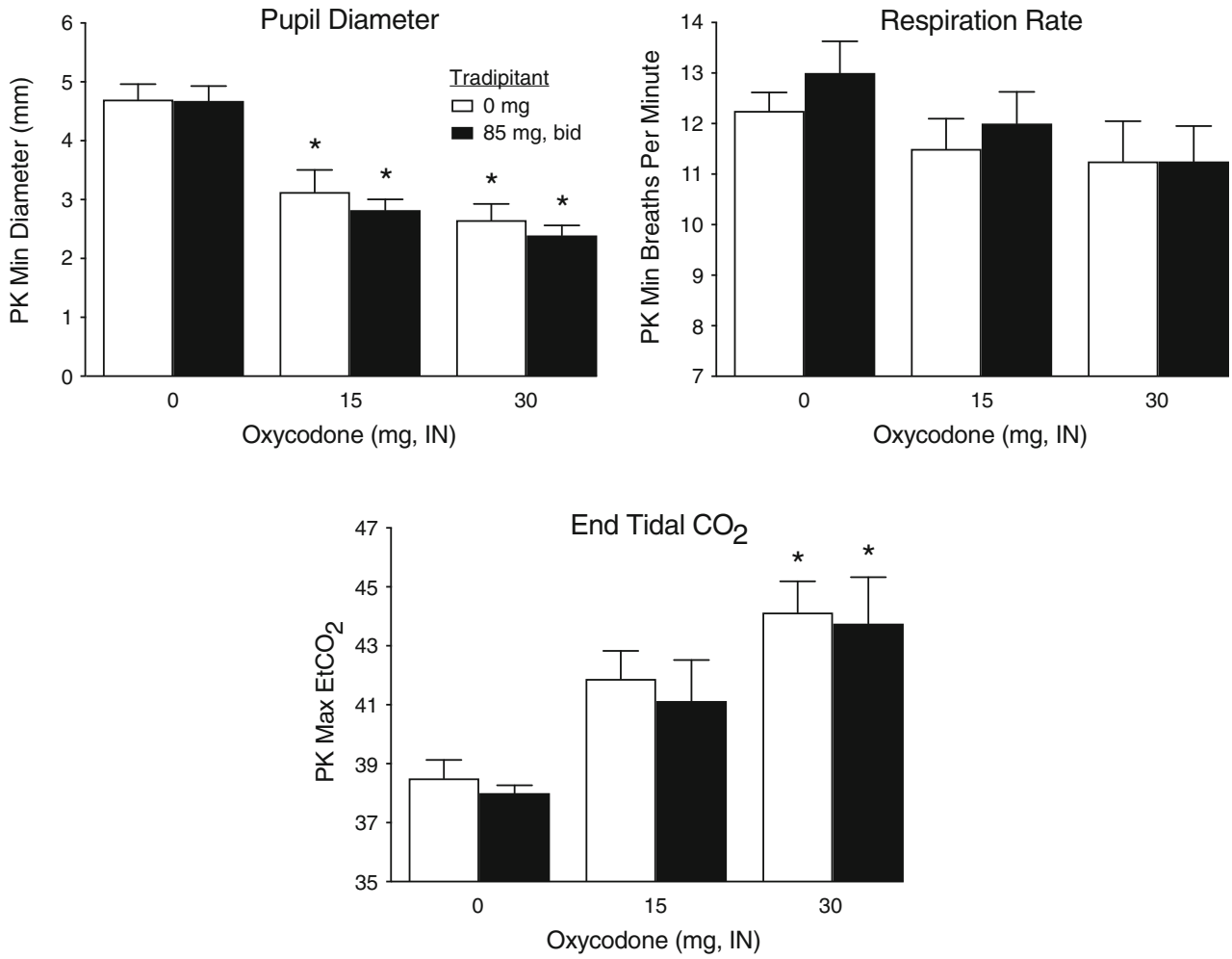

\section{Discussion}

This study examined the ability of the NK1 antagonist, tradipitant, to alter the subjective, reinforcing, physiological, and analgesic effects of oxycodone in persons with histories of recreational opioid use but without physical dependence on opioids. Intranasal oxycodone expectedly produced typical mu opioid agonist effects, including miosis, decreased
Fig. 4 Mean values $(n=8 ; \pm 1$ SEM) are shown from the selfadministration sessions for trials worked for drug (upper left), trials worked for money (upper right), money earned (lower left), and trials not worked (lower right) which are displayed as a function of tradipitant and oxycodone dose condition. There was a main effect of oxycodone for all four outcomes (trials worked for drug $\mathrm{F}_{(2,14)}=38.4, p<0.0001$; trials worked for money $\mathrm{F}_{(2,14)}=5.2$, $p=0.02$; money earned $\mathrm{F}_{(2,14)}=$ $5.2, p=0.02$; trials not worked $\left.\mathrm{F}_{(2,14)}=16.2, p=0.0002\right)$. Asterisks (*) indicate a significant difference from the placebo within tradipitant dose condition (Tukey post hoc, $p<0.05$ )
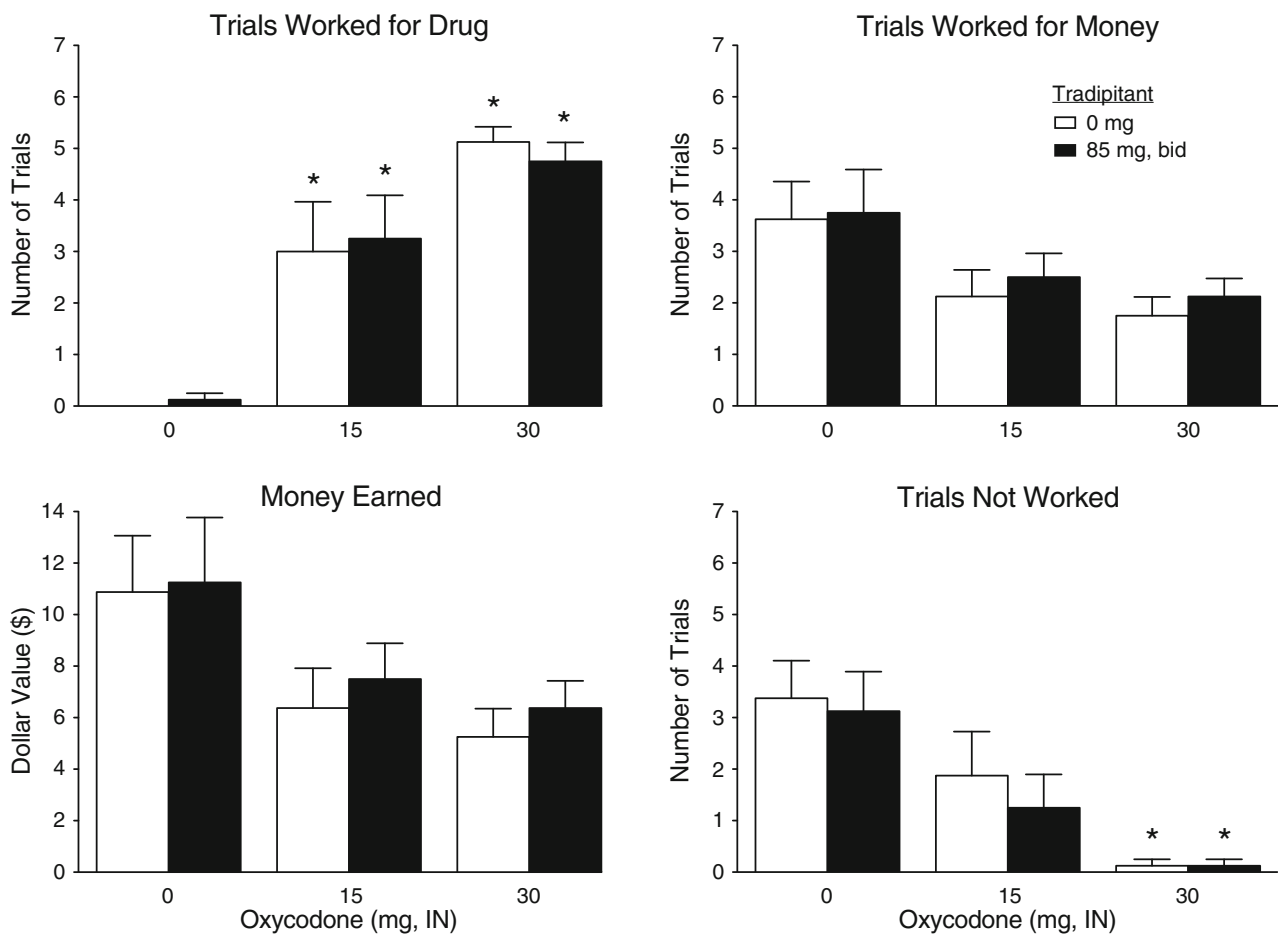
Fig. 5 Mean values $(n=8 ; \pm 1$ SEM) are shown for behavioral (threshold and tolerance) and subjective cold pressor outcomes as a function of tradipitant and oxycodone dose. Oxycodone dose is displayed on the $\mathrm{x}$-axis; tradipitant data (open symbols denote $0 \mathrm{mg} /$ bid; black symbols denote $85 \mathrm{mg} / \mathrm{bid}$ ) are shown as line functions after initiation (circles) and steady state (squares). There was a main effect of oxycodone for all outcomes (cold pressor threshold $\mathrm{F}_{(4,28)}=$ $12.9, p<0.0001$; cold pressor tolerance $\mathrm{F}_{(4,28)}=18.5, p<$ 0.0001 ; painful $\mathrm{F}_{(4,28)}=13.2, p<$ 0.0001 ; intense $\mathrm{F}_{(4,28)}=14.9, p<$ $0.0001)$

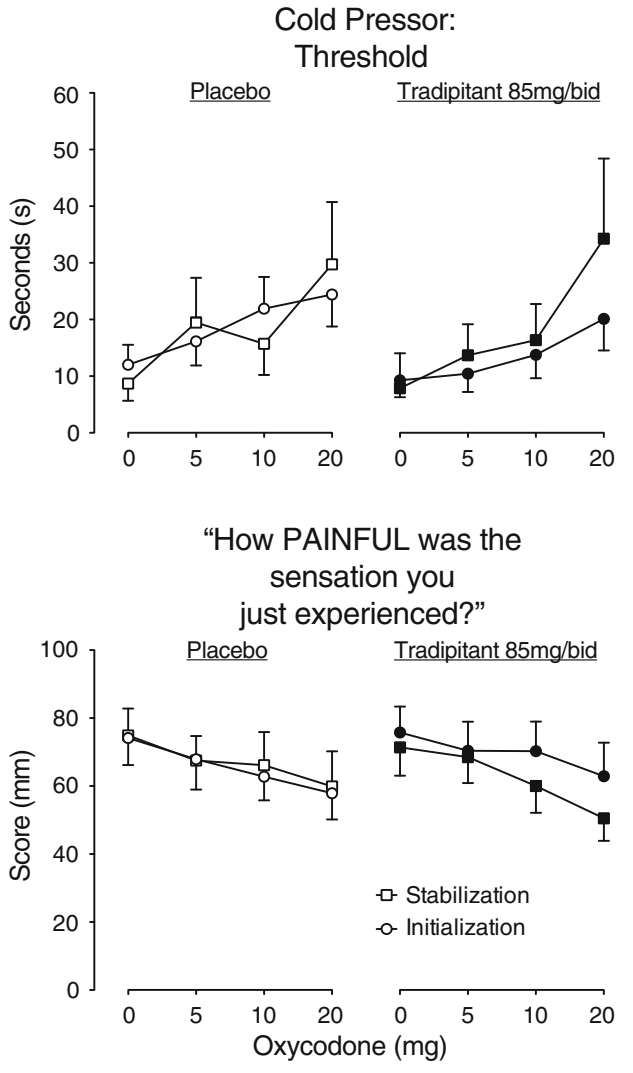

Cold Pressor: Tolerance

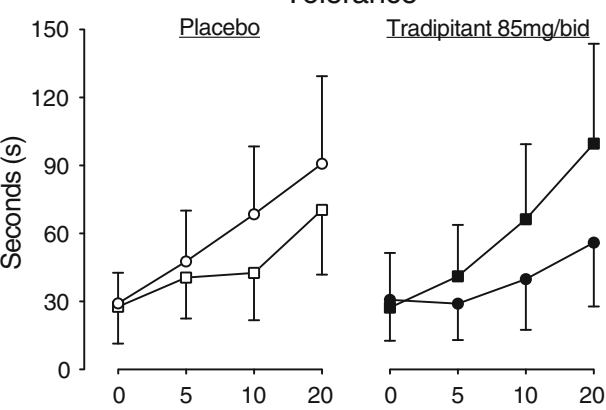

"How INTENSE was the sensation you just experienced?"

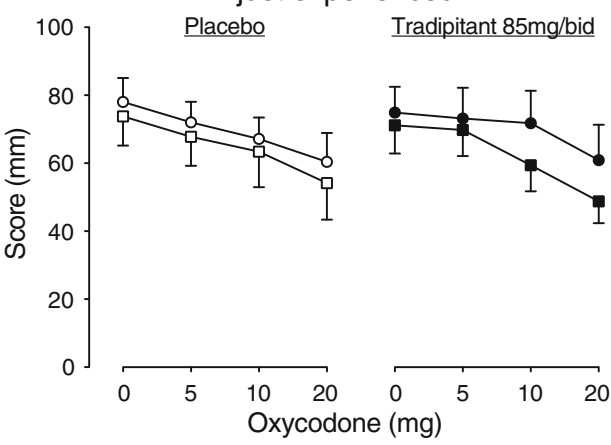

respiratory rate, analgesia and subjective effects related to euphoria; however, the vast majority of these effects were unaltered by tradipitant maintenance compared to placebo. Similarly, participants self-administered more oxycodone than placebo and demonstrated dose-related analgesia in response to oxycodone, but these too were unaltered during maintenance on active tradipitant.

Although the majority of outcomes showed no significant impact of tradipitant maintenance on oxycodone pharmacodynamics, there was a small subset of findings that suggest tradipitant was pharmacologically active and producing some direct effects. For example, ratings of desire for opioids were reduced after tradipitant compared to placebo, and the data also suggested that this reduction was greater after steady state was achieved compared to after the first active tradipitant dose. This finding needs to be considered in the context of all other outcomes as the data show that reduced "desire" (as a proxy for craving) did not lead to behavior change (i.e., no change in self-administration or abuse liability profile of oxycodone). Thus, the potential for an NK1 antagonist to be used in the treatment of opioid use disorder seems quite limited. That said, this finding could be noteworthy as it parallels at least one study from the alcohol literature. As with opioids, preclinical studies have shown that NK1 knockout mice exhibit reduced voluntary alcohol self-administration (George et al. 2008). A related clinical study then detoxified individuals with alcohol use disorder and randomly assigned to receive either tradipitant or placebo. In that study, tradipitant reduced spontaneous craving for alcohol and also attenuated craving that was experimentally provoked (George et al. 2008). However, a subsequent study examined the effect of aprepitant in individuals with alcohol use disorder and posttraumatic stress and did not replicate the finding of the NK1 antagonist reducing experimentally induced craving for alcohol (Kwako et al. 2015).

The observed pattern of findings does not support the hypothesis that an NK1 antagonist, given chronically and at doses resulting in greater than $90 \%$ receptor occupancy, can reduce the abuse liability of opioids by blunting the response to opioids as numerous preclinical studies suggest (Murtra et al. 2000; Ripley et al. 2002; Gadd et al. 2003; Barbier et al. 2013). In contrast to this body of work, previous human studies found that aprepitant actually increased measures of abuse liability when administered with either oxycodone (Walsh et al. 2013) or methadone (Jones et al. 2013). While the enhancement of opioid effects was not observed in the current study, together these reports suggest that NK1 receptor antagonism may not be a viable candidate mechanism for the treatment of opioid use disorder or opioid-sparing analgesia.

Tradipitant was selected for investigation because it is highly selective for the NK1 receptor, is not reported to produce meaningful drug-drug interactions via CYP4503A4, and has been shown to achieve $93 \pm 4 \%$ receptor occupancy in human frontal 
cortex following steady-state dosing of $100 \mathrm{mg}$ daily (Tauscher et al. 2010). The current study design also allowed for evaluation of the interaction between tradipitant and oxycodone after acute pretreatment with tradipitant and after steady-state concentrations were achieved. Given these study attributes along with the randomized, placebo-controlled within subject design, we are fairly confident that the results presented here are not due to insufficient pharmacological activity at the NK1 receptor. One limitation is the relatively small sample size; however, this did not limit the robust detection of oxycodone effects. A second limitation is that, while we enrolled females, none completed the study. While in vitro and in vivo preclinical studies provide strong evidence for relevant biological interactions between the NK1 and the opioid systems, the absence of meaningful interactions between tradipitant and oxycodone in the current study suggests that these findings may not translate to humans. In summary, tradipitant was safely tolerated alone and in combination with oxycodone in this population, but neither acute nor chronic tradipitant administration significantly altered the response to oxycodone over a broad range of measures, including subjective, physiological, reinforcement, or analgesia; these findings do not support the continued pursuit of NK1 antagonists for the treatment of opioid use disorder.

Funding This study was supported by the National Institute on Drug Abuse (R01 DA040637). Vanda Pharmaceuticals provided the active tradipitant and matched placebo capsules without charge. Vanda Pharmaceuticals had no role in the design, conduct or analysis of this project.

\section{Declarations}

Conflict of interest The authors have no direct conflicts of interest to report related to this work. Marion Coe is an employee of Pinney Associates, Inc., which provides consulting services to pharmaceutical sponsors developing medicines for pain, substance use, and other disorders.

\section{References}

Barbier E et al (2013) The NK1 receptor antagonist L822429 reduces heroin reinforcement. Neuropsychopharmacology 38:976-984. https://doi.org/10.1038/npp.2012.261

Fraser HF, Van Horn GG, Martin WR, Wolbach AB, Isbell H (1961) Methods for evaluating addiction liability. (A) attitude of opiate addicts toward opiate-like drugs, (B) a short-term "direct" addiction test. J Pharmacol Exp Ther 133:371-378

Gadd CA, Murtra P, De Felipe C, Hunt SP (2003) Neurokinin-1 receptorexpressing neurons in the amygdala modulate morphine reward and anxiety behaviors in the mouse. The Journal of Neuroscience 23: $8271-8280$

George DT, Gilman J, Hersh J, Thorsell A, Herion D, Geyer C, Peng X, Kielbasa W, Rawlings R, Brandt JE, Gehlert DR, Tauscher JT, Hunt SP, Hommer D, Heilig M (2008) Neurokinin 1 receptor antagonism as a possible therapy for alcoholism. Science 319:1536-1539
Hargreaves R (2002) Imaging substance P receptors (NK1) in the living brain using positron emission tomography. J Clin Psychiatry 63:18-24

Jones JD, Speer T, Comer SD, Ross S, Rotrosen J, Reid MS (2013) Opioid-like effects of the neurokinin 1 antagonist aprepitant in patients maintained on and briefly withdrawn from methadone. Am J Drug Alcohol Abuse 39:86-91. https://doi.org/10.3109/00952990. 2012.762372

Kwako LE et al (2015) The neurokinin-1 receptor antagonist aprepitant in co-morbid alcohol dependence and posttraumatic stress disorder: a human experimental study. Psychopharmacology (Berl) 232:295304. https://doi.org/10.1007/s00213-014-3665-4

Majumdar AK et al (2003) Effects of aprepitant on cytochrome P450 3A4 activity using midazolam as a probe. Clin Pharmacol Ther 74:150 156. https://doi.org/10.1016/S0009-9236(03)00123-1

Merck \& Co. I (2021) Emend® package insert. https://www.accessdata. fda.gov/drugsatfda_docs/label/2015/207865lbl.pdf. Accessed 5 Jan 2021

Murtra P, Sheasby AM, Hunt SP, De Felipe C (2000) Rewarding effects opiates are absent in mice lacking the receptor for substance $\mathrm{P}$. Nature 405:180-183

Preston KL, Bigelow GE, Liebson IA (1987) Comparative evaluation of morphine, pentacozine and ciramadol in postaddicts. J Pharmacol Exp Ther 240:900-910

Ratti E, Bellew K, Bettica P, Bryson H, Zamuner S, Archer G, Squassante L, Bye A, Trist D, Krishnan KR, Fernandes S (2011) Results from 2 randomized, double-blind, placebo-controlled studies of the novel NK1 receptor antagonist casopitant in patients with major depressive disorder. J Clin Psychopharmacol 31:727-733. https://doi.org/ 10.1097/JCP.0b013e31823608ca

Ripley TL, Gadd CA, De Felipe C, Hunt SP, Stephens DN (2002) Lack of self-administration and behavioural sensitisation to morphine, but not cocaine, in mice lacking NK1 receptors. Neuropharmacology 43:1258-1268

Robinson JE, Fish EW, Krouse MC, Thorsell A, Heilig M, Malanga CJ (2012) Potentiation of brain stimulation reward by morphine: effects of neurokinin-1 receptor antagonism. Psychopharmacology 220: 215-224. https://doi.org/10.1007/s00213-011-2469-z

Sanchez RI et al (2004) Cytochrome P450 3A4 is the major enzyme involved in the metabolism of the substance $\mathrm{P}$ receptor antagonist aprepitant. Drug Metab Dispos 32:1287-1292. https://doi.org/10. 1124/dmd.104.000216

Singewald N et al (2008) Modulation of basal and stress-induced amygdaloid substance $\mathrm{P}$ release by the potent and selective NK1 receptor antagonist L-822429. J Neurochem 106:2476-2488. https://doi.org/ 10.1111/j.1471-4159.2008.05596.x

Tauscher J et al (2010) Development of the 2nd generation neurokinin-1 receptor antagonist LY686017 for social anxiety disorder. Eur Neuropsychopharmacol 20:80-87. https://doi.org/10.1016/j. euroneuro.2009.10.005

Walsh SL, Heilig M, Nuzzo PA, Henderson P, Lofwall MR (2013) Effects of the NK1 antagonist, aprepitant, on response to oral and intranasal oxycodone in prescription opioid abusers. Addict Biol 18: 332-343. https://doi.org/10.1111/j.1369-1600.2011.00419.x

Walsh SL, Nuzzo PA, Lofwall MR, Holtman JR Jr (2008) The relative abuse liability of oral oxycodone, hydrocodone and hydromorphone assessed in prescription opioid abusers. Drug Alcohol Depend 98: 191-202. https://doi.org/10.1016/j.drugalcdep.2008.05.007

Zamuner S et al (2012) A pharmacokinetic PET study of NK(1) receptor occupancy. Eur J Nucl Med Mol Imaging 39:226-235. https://doi. org/10.1007/s00259-011-1954-2

Publisher's note Springer Nature remains neutral with regard to jurisdictional claims in published maps and institutional affiliations. 